\title{
Multiple Sampling from the Central Veins with their Tributaries can Detect Bilateral Hyperaldosteronism with a Cortisol-Producing Adenoma in a Hypertensive Patient
}

\author{
${ }^{1}$ Endocrinology and Diabetes Center, Yokohama Rosai Hospital, Yokohama, Japan \\ ${ }^{2}$ Department of Radiology, Yokohama Rosai Hospital, Yokohama, Japan \\ ${ }^{3}$ Departments of Urology, School of Medicine, Keio University, Tokyo, Japan \\ ${ }^{4}$ Department of Urology, Tachikawa Hospital, Tokyo, Japan \\ ${ }^{5}$ Department of Biochemistry, School of Medicine, Keio University, Tokyo, Japan
}

Ikki Sakuma ${ }^{1}$, Jun Saito ${ }^{1}$, Yoko Matsuzawa ${ }^{1}$, Masao Omura ${ }^{1}$, Seiji Matsui ${ }^{2}$, Koshiro Nishimoto ${ }^{3,4}$, Kuniaki Mukai ${ }^{5}$ and Tetsuo Nishikawa $^{{ }^{*}}$

"Corresponding author: Tetsuo Nishikawa, Endocrinology and Diabetes Center, Yokohama Rosai Hospital, 3211 Kozukue-cho, Kohoku-ku, Yokohama City, Kanagawa 222-0036, Japan, Tel: +81-454748111; Fax: +81-454748323; E-mail: tetsuon@yokohamah.rofuku.go.jp

Received date: April 15, 2014, Accepted date: June 11, 2014, Published date: June 18, 2014

Copyright: ( 2014 Sakuma I, et al. This is an open-access article distributed under the terms of the Creative Commons Attribution License, which permits unrestricted use, distribution, and reproduction in any medium, provided the original author and source are credited.

\begin{abstract}
A 52-year old woman was admitted to our hospital for evaluation of left adrenal incidenataloma. Endocrinological examination showed Cushing's syndrome (CS) complicated with masked primary aldosteronism (PA). On the other hand, multiple sampling from the central veins and one or two tributaries of the adrenal veins before and after ACTH-stimulation (multiple AVS) clearly revealed bilateral hyperaldosteronism with excess cortisol secretion from the left adrenal. Thus, we diagnosed this case as CS due to left adrenal tumor with bilateral hyperaldosteronism, and left adrenalectomy was done. Immunohistochemical analysis of the removed left adrenal showed cortisol-producing adenoma and multiple aldosterone-producing cell clusters (APCCs) expressing CYP11B2 within the attached adrenal. Bilateral PA is mostly diagnosed as idiopathic hyperaldosteronism (IHA). IHA has not been examined enough pathologically. We first describe here a possible involvement of APCCs inducing hyperaldosteronism in a case of bilateral PA with a cortisol-producing-adenoma.
\end{abstract}

Keywords: Idiopathic hyperaldosteronism; Aldosterone-producing cell clusters; Adrenal vein sampling; Cushing's syndrome

\section{Introduction}

Here, we describe a case of left adrenal incidentaloma in a patient with Cushing's syndrome (CS) simultaneously complicating hyperaldosteronism. In US and Japan, a screening program for primary aldosteronism (PA), which is a major cause of endocrine hypertension, was recently introduced [1,2]. Aldosterone producing adenoma (APA) and idiopathic hyperaldosteronism (IHA) are the main causes of PA. APA usually responds to unilateral adrenalectomy, which corrects hyperaldosteronism and can attenuate hypertension. The medical management of IHA is generally recommended. Therefore IHA has not been examined enough pathologically. On the other hands, Nishimoto et al. have recently established an immunohistochemical technique for detecting CYP11B1 and CYP11B2 and suggested that it would allow the confirmatory pathological diagnosis of APA [3]. They detected areas of variegated zonation, in which the abnormal zones consisted of CYP11B2expressing subcapsular cell clusters, which are termed as aldosteroneproducing cell clusters (APCCs). They proposed that in the human adrenal cortex aldosterone production is constitutive in APCC and inducible in the zona glomerulosa of the conventional tissue [3]. In this case, results of multiple sampling from the central veins and one or two tributaries of the adrenal veins before and after ACTHstimulation (multiple AVS) and immunohistochemistry of steroidogenic enzymes suggested multiple APCCs, possibly inducing bilateral hyperaldosteronism. We first describe a possible involvement of APCCs-induced hyperaldosteronism in bilateral PA with a cortisolproducing adenoma.

\section{Case Report}

A 52-year-old woman was admitted to Yokohama Rosai Hospital in order to have her left adrenal incidentaloma and hypertension evaluated. A few months before her referral to our hospital, abdominal $\mathrm{CT}$, which was performed due to abdominal pain, detected a left adrenal tumor. Abdominal pain gradually disappeared and was thought to be caused by gastroenteritis. She had previously been diagnosed with hypertension and had started taking anti-hypertensive medication (nifedipine) at the age of 50. A physical examination revealed that she measured $153 \mathrm{~cm}$ in height and weighed $39 \mathrm{~kg}$, indicating emaciation. Her blood pressure was $183 / 104 \mathrm{mmHg}$ even under taking a antihypertensive drug. She displayed some cushingoid features such as thin skin and hirsutism. Laboratory examination showed dyslipidemia and impaired glucose tolerance. $75 \mathrm{~g}$ oral glucose tolerance test showed diabetic pattern $(0 \mathrm{~min}, 74 \mathrm{mg} / \mathrm{dl} ; 120 \mathrm{~min}, 214$ $\mathrm{mg} / \mathrm{dl}$ ). A bone X-ray and dual-energy X-ray absorptiometry (DEXA) of lumbar spine revealed osteoporosis (T-score: -3.6).

An endocrinological examination (Table 1) revealed that the circadian rhythm of the patient's hypothalamic-pituitary-adrenal axis had been disturbed by persistently suppressed ACTH levels. Her urine free cortisol level was elevated to 252 (normal range: 11.2 80.3) $\mu \mathrm{g} /$ day. However, her cortisol level was not suppressed $(14.9 \mu \mathrm{g} / \mathrm{dL})$ by the administration of $1 \mathrm{mg}$ dexamethasone. Abdominal CT with 
Citation: Sakuma I, Saito J, Matsuzawa Y, Omura M, Matsui S, et al. (2014) Multiple Sampling from the Central Veins with their Tributaries can Detect Bilateral Hyperaldosteronism with a Cortisol-Producing Adenoma in a Hypertensive Patient. J Steroids Hormon Sci 5: 135. doi: $10.4172 / 2157-7536.1000135$

Page 2 of 5

contrast medium demonstrated a left adrenal tumor $(30 \mathrm{~mm}$ in a diameter), while the right adrenal gland was found to be atrophic (Figure 1A and 1B). Plain CT also showed the left adrenal tumor of 33 Hounsfield units. In ${ }^{131}$ I-adosterol scintigraphy, the radioisotope was taken up by the left adrenal tumor but not by the contralateral adrenal gland (Figure 1C). On the basis of these results, she was diagnosed as CS [4].

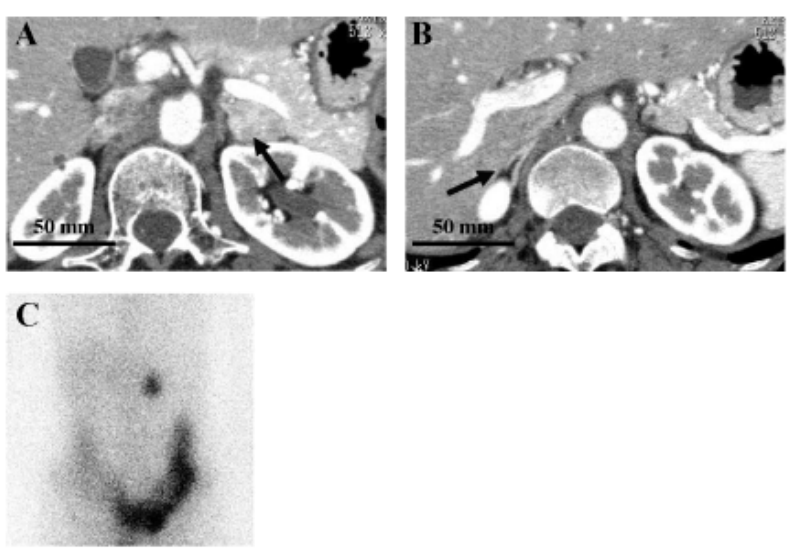

Figure 1: Abdominal CT showed (A) the left adrenal adenoma indicated by arrow, and (B) the right atrophic adrenal gland indicated by arrow. (C) ${ }^{131} \mathrm{I}$-adosterol scintigraphy. Increased uptake of radioisotope accumulation in left adrenal tumor with suppression of right adrenal was noted.

\begin{tabular}{|c|c|c|c|c|}
\hline \multicolumn{5}{|l|}{ Serum } \\
\hline $\mathrm{Na}$ & 144 & $\mathrm{mEq} / \mathrm{L}$ & & \\
\hline $\mathrm{K}$ & 3.8 & $\mathrm{mEq} / \mathrm{L}$ & & \\
\hline ACTH & $<1.0$ & $\mathrm{pg} / \mathrm{mL}$ & & \\
\hline CS & 12.4 & $\mu \mathrm{g} / \mathrm{dL}$ & & \\
\hline PRA & 0.5 & $\mathrm{ng} / \mathrm{mL} / \mathrm{hr}$ & & \\
\hline PAC & 133 & $\mathrm{pg} / \mathrm{mL}$ & & \\
\hline DHEA-S & 20 & $\mu \mathrm{g} / \mathrm{dL}$ & & \\
\hline \multicolumn{5}{|l|}{ Urine } \\
\hline $\mathrm{CCr}$ & 107.8 & $\mathrm{~mL} / \mathrm{min}$ & & \\
\hline $\mathrm{Na}$ & 140.6 & $\mathrm{mEq} / \mathrm{day}$ & & \\
\hline $\mathrm{K}$ & 52.3 & $\mathrm{mEq} /$ day & & \\
\hline free cortisol & 252 & $\mu \mathrm{g} / \mathrm{day}$ & & \\
\hline aldosterone & 5.4 & $\mu \mathrm{g} / \mathrm{day}$ & & \\
\hline \multicolumn{5}{|c|}{ Diural rhythm and dexamethasone suppression test } \\
\hline & $8: 00$ & $23: 00$ & Dex $1 \mathrm{mg}$ & \\
\hline ACTH $(\mathrm{pg} / \mathrm{mL})$ & $<1.0$ & $<1.0$ & $<1.0$ & \\
\hline$C S(\mu \mathrm{g} / \mathrm{dL})$ & 12.4 & 11.8 & 14.9 & \\
\hline
\end{tabular}

\begin{tabular}{|c|c|c|c|c|}
\hline PRA (ng/mL/hr) & 0.1 & $<0.1$ & & \\
\hline PAC $(p g / m L)$ & 70 & 74 & 65 & \\
\hline \multicolumn{5}{|c|}{ CRH stimulation test, $100 \mu \mathrm{g}$ iv } \\
\hline & 0 min & $30 \mathrm{~min}$ & $60 \mathrm{~min}$ & $120 \mathrm{~min}$ \\
\hline ACTH $(p g / m L)$ & $<1.0$ & 1.6 & $<1.0$ & 1.6 \\
\hline $\mathrm{CS}(\mu \mathrm{g} / \mathrm{dL})$ & 13.3 & 16.5 & 15.4 & 13.6 \\
\hline \multicolumn{5}{|c|}{ ACTH stimulation test, $250 \mu \mathrm{g}$ iv } \\
\hline & 0 min & $30 \mathrm{~min}$ & $60 \mathrm{~min}$ & \\
\hline $\mathrm{CS}(\mu \mathrm{g} / \mathrm{dL})$ & 14.6 & 29.4 & 35.1 & \\
\hline PAC $(p g / m L)$ & 128 & 384 & 408 & \\
\hline PRA (ng/mL/hr) & 0.4 & & & \\
\hline \multicolumn{5}{|c|}{$\begin{array}{l}\text { Captopril test, } 50 \mathrm{mg} \\
\text { po }\end{array}$} \\
\hline & $0 \mathrm{~min}$ & $90 \mathrm{~min}$ & & \\
\hline PRA (ng/mL/hr) & 0.2 & 0.6 & & \\
\hline PAC $(\mathrm{pg} / \mathrm{mL})$ & 43 & 56 & & \\
\hline ARR & 215 & 93.3 & & \\
\hline \multicolumn{5}{|c|}{ Saline infusion test, $2 \mathrm{~L}$ div } \\
\hline & & $240 \mathrm{~min}$ & & \\
\hline PRA (ng/mL/hr) & & 0.3 & & \\
\hline PAC $(\mathrm{pg} / \mathrm{mL})$ & & 71 & & \\
\hline \multicolumn{5}{|c|}{ Frosemide upright test, $20 \mathrm{mg}$ iv } \\
\hline & & $240 \mathrm{~min}$ & & \\
\hline PRA (ng/mL/hr) & & 2.1 & & \\
\hline PAC $(p g / m L)$ & & 358 & & \\
\hline \multicolumn{5}{|l|}{ Multiple AVS } \\
\hline & \multicolumn{2}{|l|}{ RAV } & \multicolumn{2}{|l|}{ LAV } \\
\hline & $\begin{array}{l}\text { PAC } \\
(\mathrm{pg} / \mathrm{mL})\end{array}$ & $\mathrm{CS}(\mu \mathrm{g} / \mathrm{dL})$ & $\begin{array}{l}\text { PAC } \\
(\mathrm{pg} / \mathrm{mL})\end{array}$ & $\operatorname{CS}(\mu \mathrm{g} / \mathrm{dL})$ \\
\hline Central & 36200 & 200 & 23400 & 1100 \\
\hline Inferior & 29500 & 172 & 21800 & 853 \\
\hline Lateral & 33000 & 197 & 4490 & 1390 \\
\hline
\end{tabular}

Table 1: Results of endocrine function tests before surgical treatment

ARR: Aldosterone Renin Ratio; AVS: Adrenal Vein Sampling; CS: Cortisol; Dex: Dexamethasone; LAV: Left Adrenal Vein; PAC: Plasma Aldosterone Concentration; PRA: Plasma Renin Activity; RAV: Right Adrenal Vein.

Interestingly, in addition to hyper production of cortisol, this case showed slightly elevated PAC of $128 \mathrm{pg} / \mathrm{mL}$, suppressed PRA of 0.4 
Citation: Sakuma I, Saito J, Matsuzawa Y, Omura M, Matsui S, et al. (2014) Multiple Sampling from the Central Veins with their Tributaries can Detect Bilateral Hyperaldosteronism with a Cortisol-Producing Adenoma in a Hypertensive Patient. J Steroids Hormon Sci 5: 135. doi: $10.4172 / 2157-7536.1000135$

Page 3 of 5

$\mathrm{ng} / \mathrm{mL} / \mathrm{hr}$, and elevated aldosterone renin ratio (ARR) of 320 , suggesting the possibility of primary aldosteronism (PA) according to the guidelines [2]. Therefore we investigated whether or not PA was also associated. Captopril challenge test showed suppression of ARR from 215 to 93.3, while PAC was not suppressed to less than $60 \mathrm{pg} / \mathrm{mL}$ by saline infusion test $(71 \mathrm{pg} / \mathrm{mL})$. ACTH stimulation test with 0.25 $\mathrm{mg}$ cosyntropin also demonstrated a marked increase in PAC to 408 $\mathrm{pg} / \mathrm{mL}$ in the present case. These results indicated a high possibility of the autonomous secretion of aldosterone.

Left adrenal tumor was expected to cause CS. However, subtype of PA in this case was considered a variety of adrenal pathologies, such as IHA, left adrenal tumor co-producing aldosterone and cortisol, or double adenomas which consist of left cortisol producing adenoma and micro APA at ipsilateral bilateral or contralateral side. Multiple AVS was performed to determine precisely the subtype of PA because therapeutic approach may differ depending on the disease subtype (Table 1). The central, inferior tributary, and lateral tributary veins of the right adrenal displayed aldosterone levels of 36200, 29500, and $33000 \mathrm{pg} / \mathrm{mL}$, and cortisol levels of $200,172,197 \mu \mathrm{g} / \mathrm{dL}$ respectively. On the other hands, the central, superior tributary, and lateral tributary veins of the left adrenal exhibited aldosterone levels of 23400, 21800 , and $4490 \mathrm{pg} / \mathrm{mL}$, and cortisol levels of 1100,853 , and 1390 $\mu \mathrm{g} / \mathrm{dL}$ respectively. Venography of the left lateral tributary detected tumor staining. CT revealed an adrenal tumor attached to the left lateral adrenal gland. Therefore, we assumed that the left lateral tributary was connected to the tumor. Cortisol level in the left superior tributary was not sufficiently suppressed compared to that in the lateral tributary, indicating hormone secretion from the tumor partially flowed into the superior tributary. Evaluation criteria of hyperaldosteronism based on the aldosterone/cortisol ratio was not used because of autonomous cortisol secretion. We could simply detect the presence of hyperaldosteronism by analysing absolute values of aldosterone in each central and tributary vein. We reported that the cut-off value of hyperaldosteronism was 14,000 pg/mL (adrenal vein PAC after ACTH stimulation) [2]. The left lateral tributary displayed cortisol hypersecretion but not hyperaldosteronism. On the basis of these results, we judged that the left adrenal tumor secreted cortisol without hyperaldosteronism and that bilateral PA due to IHA was responsible for the patient's hyperaldosteronism. In order to control excessive secretion of cortisol (but not that of aldosterone), we performed laparoscopic left adrenalectomy. Left adrenal tumor $28 \times 23 \times 10 \mathrm{~mm}$, and was yellowish in color with lipofuscin granules. A histopathological examination showed that it was composed of compact cells with eosinophilic cytoplasm. An immunohistochemical examination of the tumor detected immunoreactivity to CYP17, $3 \beta \mathrm{HSD}$ and CYP11B1, but not to CYP11B2 (Figure 2A-2C). The adjacent adrenal cortex was grossly atrophic, which was consistent with CS. Multiple CYP11B2-expressing sub capsular cell clusters, which were indicative of APCC, were detected diffusely in the adjacent adrenal cortex (Figure 2D and 2G). 33HSD was also expressed in the APCCs (Figure 2E). CYP17 was not expressed in the APCCs (Figure 2F). After left adrenalectomy, a second endocrinological examination was performed (Table 2). As a result, it was found that her cortisol levels had markedly decreased, indicating cure of Cushing's syndrome. And her aldosterone levels had halved. An ACTH stimulation test produced an increase in her PAC to $241 \mathrm{pg} / \mathrm{mL}$. Her blood pressure returned to within the normal range soon after surgery without the administration of any medication. The patient was discharged uneventfully at 16th postoperative day under supplementation of 10 $\mathrm{mg}$ of hydrocortisone. Hydrocortisone was gradually tapered. And 1- year after surgery, a third endocrinological examination was performed (Table 3). As a result, ACTH and cortisol levels improved, compared to just after surgery. On the other hands, aldosterone response to ACTH stimulation fell within normal range, indicating remission of IHA.

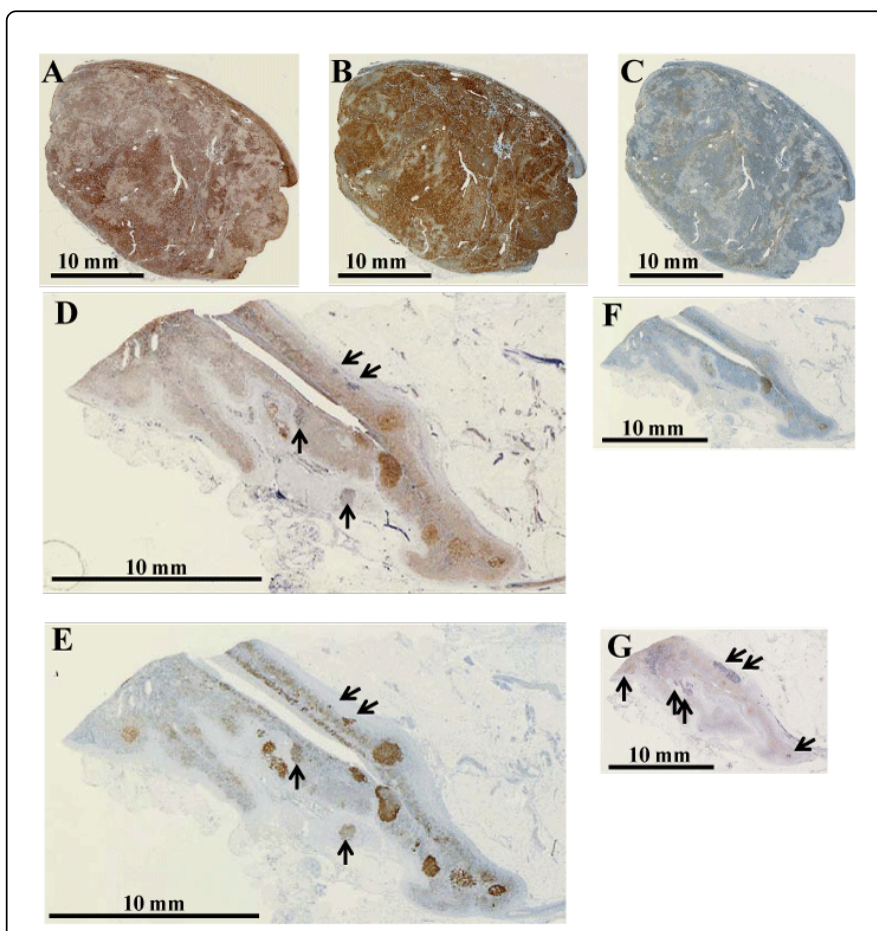

Figure 2: Pathological findings of cortisol-producing lesions in left adrenal. Sections prepared from adrenal gland of the present case were examined histologically and immunohistochemically. (A) Double-immunostaining for CYP11B2 (blue) and CYP11B1 (brown). (B) Immunohistochemistry of $3 \beta$-HSD (brown). (C) Immunohistochemistry of CYP17 (brown). Panels (D) (E) (F) and $(G)$ are showing histopathology of the attached adrenal tissue. Panel (D) and (G), Double-immunostaining of the adjacent adrenal for CYP11B2 (blue) and CYP11B1 (brown). There were multiple APCCs (indicated by arrow) at the adjacent adrenal cortex. Panel (E), Immunohistochemistry of the adjacent for $3 \beta$-HSD (brown). $3 \beta$ HSD was expressed in the APCCs (indicated by arrow). Panel (F), Immunohistochemistry of the adjacent for CYP17 (brown). CYP17 was not expressed in the APCCs. Nuclei were counterstained with hematoxylin after immunostaining for $3 \beta$ HSD and CYP17.

\section{Discussion}

We described a case with CS due to left cortisol producing adenoma and bilateral hyperaldosteronism. We preoperatively diagnosed the patient with bilateral hyperaldosteronism based on the results of multiple AVS and confirmed multiple APCCs as the cause of her excess aldosterone levels via immunohistochemical examinations of her steroidogenic enzyme expression.

Patients with concurrent CS and PA have been reported in several studies [5-8]. Pediatis et al. reported that PA associated with hypercortisolism occured $12.1 \%$ in 83 adrenal incidentalomas [7]. 
Citation: Sakuma I, Saito J, Matsuzawa Y, Omura M, Matsui S, et al. (2014) Multiple Sampling from the Central Veins with their Tributaries can Detect Bilateral Hyperaldosteronism with a Cortisol-Producing Adenoma in a Hypertensive Patient. J Steroids Hormon Sci 5: 135. doi: $10.4172 / 2157-7536.1000135$

Page 4 of 5

Hiraishi et al. detected PA complicated with subclinical CS in 8 of 38 PA patients (21\%) [9]. A recent study suggested that the ACTH test can be useful in PA subtype diagnosis [10]. In PA with CS, hyperaldosteronism could be masked because ACTH suppressed by CS may decrease aldosterone production. ACTH stimulation test was reported to be useful for detecting masked hyperaldosteronism with CS [8]. In our case, clinical signs and endocrinological data suggested CS possibly associated with masked PA before performing AVS. PA manifests as several subtypes with different biochemical and pathological characteristics, with APA and IHA accounting for most cases [11]. Concurrent CS and PA cases also could be classified into several subtypes of PA [9]. It is well known that conventional AVS could detect the laterality of hyperaldosteronism. Moreover, multiple AVS could easily detect the portions of localized (APA) or diffuse adrenal lesions (IHA) compared to conventional AVS [12]. By multiple AVS, we preoperatively diagnosed the present case as CS due to a left cortisol-producing adenoma associated with bilateral hyperaldosteronism (IHA). Immunohistochemistry of the tumor detected immunoreactivity to CYP17, 3ßHSD and CYP11B1, but not to CYP11B2. These findings suggested that the tumor secreted cortisol and did not be involved in hyperaldosteronism. On the other hands, immunohistochemistry of the adjacent atrophic adrenal cortex detected multiple APCCs. Immunohistochemistry of APCCs detected immunoreactivity to $3 \beta \mathrm{HSD}$ and CYP11B2, but not to CYP17 and CYP11B1, indicating that those APCCs could mainly produce aldosterone. Some APCCs were relatively large (about $2 \mathrm{~mm}$ ), which was estimated to be etiology of hyperaldosteronism. Furthermore, bilateral hyperaldosteronism detected by multiple AVS was possibly caused by APCCs in bilateral adrenals even though right adrenalectomy was not performed yet. On the other hands, Nishimoto et al. suggested that APCCs were found in some cases of normal adrenals and nontumor portions of cortisol producing adenoma [3]. Number of APCCs in our case was not especially higher than that in the previous paper. It has not been elucidated sufficiently that how many and large APCCs cause hyperaldosteronism. Further study should be done to investigate this issue.

Screening for PA is spreading in Japan, and hence, the number of cases of IHA has been on the increase. According to our findings, the incidence of IHA among PA patients was 26 60\% [13,14]. Pathologically, adrenal glands in IHA are characterized by diffuse hyperplasia involving cells that resemble those found in the normal zona glomerulosa without nodules [15]. However, most IHA cases are treated with non-surgical methods because unilateral adrenalectomy has been considered ineffective [16]. Therefore, there is insufficient pathological information about IHA. The findings we obtained with multiple AVS and immunohistochemistry suggest that multiple APCCs can cause bilateral hyperaldosteronism. One-year after left adrenalectomy, PA interestingly remitted. We do not fully explain the reason why unilateral adrenalectomy could improve hyperaldosteronism in this case. It is possible to consider that excess cortisol production might affect intra-adrenal renin-angiotensin system, resulting in high aldosterone synthesis induced by locally produced angiotensin II. Thus, it is speculated that APCC existed in adrenal tissues of IHA could produce excess amounts of aldosterone stimulated by cortisol-induced intra-adrenal renin-angiotensin system in this case. On the other hand, unilateral adrenalectomy was reported to be effective on reducing blood pressure and PAC in some case with IHA, not associating with Cushing syndrome $[17,18]$. Therefore, we need to clarify the effectiveness of unilateral adrenalectomy for the patients with IHA. In conclusion, we first describe here a possible involvement of APCCs-induced hyperaldosteronism in a case of bilateral PA with a cortisol-producing adenoma. It has not been elucidated sufficiently that how many and large APCCs cause hyperaldosteronism. Furthermore we should perform pathophysiological investigations for this issue by multiple AVS and immunohistochemistry.

\section{Acknowledgements}

This work was partly supported by the Grant for Research on Intractable Diseases provided from the Japanese Ministry of Health, Labour and Welfare.

We thank Mr. Shinya Sasai, Ms. Yuka Yokomichi, and Ms Hitomi Shimizu in the Department of Pathology, Tachikawa Hospiatal for excellent technical assistance with immunohistochemistry.

\section{References}

1. Funder JW, Carey RM, Fardella C, Gomez-Sanchez CE, Mantero F, et al. (2008) Case detection, diagnosis, and treatment of patients with primary aldosteronism: an endocrine society clinical practice guideline. J Clin Endocrinol Metab. 93: 3266-3281.

2. Nishikawa T, Omura M, Satoh F, Shibata H, Takahashi K, et al. (2011) Guidelines for the diagnosis and treatment of primary aldosteronism-the Japan Endocrine Society 2009. Endocr J 58: 711-721.

3. Nishimoto K, Nakagawa K, Li D, Kosaka T, Oya M, et al. (2010) Adrenocortical zonation in humans under normal and pathological conditions. J Clin Endocrinol Metab 95: 2296-2305.

4. Nieman LK, Biller BM, Findling JW, Newell-Price J, Savage MO, et al. (2008) The diagnosis of Cushing's syndrome: an Endocrine Society Clinical Practice Guideline. J Clin Endocrinol Metab 93: 1526-1540.

5. Morimoto R, Kudo M, Murakami O, Takase K, Ishidoya S, et al. (2011) Difficult-to-control hypertension due to bilateral aldosterone-producing adrenocortical microadenomas associated with a cortisol-producing adrenal macroadenoma. J Hum Hypertens. 25: 114-121.

6. Onoda N, Ishikawa T, Nishio K, Tahara H, Inaba M, et al. (2009) Cushing's syndrome by left adrenocortical adenoma synchronously associated with primary aldosteronism by right adrenocortical adenoma: report of a case. Endocr J. 56: 495-502.

7. Piaditis GP, Kaltsas GA, Androulakis II, Gouli A, Makras P, et al. (2009) High prevalence of autonomous cortisol and aldosterone secretion from adrenal adenomas. Clin Endocrinol (Oxf) 71: 772-778.

8. Kukidome D, Miyamura N, Sakakida K, Shimoda S, Shigematu Y, et al. (2012) A Case of Cortisol Producing Adrenal Adenoma Associated with a Latent Aldosteronoma: Usefulness of the ACTH Loading Test for the Detection of Covert Aldosteronism in Overt Cushing Syndrome. Internal Medicine 51: 395-400.

9. Hiraishi K, Yoshimoto T, Tsuchiya K, Minami I, Doi M, et al. (2011) Clinicopathological features of primary aldosteronism associated with subclinical Cushing's syndrome. Endocr J 58: 543-551.

10. Sonoyama T, Sone M, Miyashita K, Tamura N, Yamahara K, et al. (2011) Significance of adrenocorticotropin stimulation test in the diagnosis of an aldosterone-producing adenoma. J Clin Endocrinol Metab 96: 2771-2778.

11. Somlóová Z, Widimský J Jr, Rosa J, Wichterle D, Strauch B, et al. (2010) The prevalence of metabolic syndrome and its components in two main types of primary aldosteronism. J Hum Hypertens 24: 625-630.

12. Omura M, Saito J, Matsuzawa Y, Nishikawa T (2011) Supper-selective ACTH-stimulated adrenal vein sampling is necessary for detecting precisely functional state of various lesions in unilateral and bilateral adrenal disorders, inducing primary aldosteronism with subclinical Cushing's syndrome. Endocr J. 58: 919-920.

13. Omura M, Sasano H, Saito J, Yamaguchi K, Kakuta Y, et al. (2006) Clinical characteristics of aldosterone-producing microadenoma, 
Citation: Sakuma I, Saito J, Matsuzawa Y, Omura M, Matsui S, et al. (2014) Multiple Sampling from the Central Veins with their Tributaries can Detect Bilateral Hyperaldosteronism with a Cortisol-Producing Adenoma in a Hypertensive Patient. J Steroids Hormon Sci 5: 135. doi: $10.4172 / 2157-7536.1000135$

Page 5 of 5

macroadenoma, and idiopathic hyperaldosteronism in 93 patients with primary aldosteronism. Hypertens Res. 29: 883-889.

14. Young WF (2007) Primary aldosteronism: renaissance of a syndrome. Clin Endocrinol (Oxf) 66: 607-618.

15. Novitsky YW, Kercher KW, Rosen MJ, Cobb WS, Jyothinagaram S, et al. (2005) Clinical outcomes of laparoscopic adrenalectomy for lateralizing nodular hyperplasia. Surgery 138: 1009-1016.

16. Jeck T, Weisser B, Mengden T, Erdmenger L, Grune S, et al. (1994) Primary aldosteronism: difference in clinical presentation and long-term follow-up between adenoma and bilateral hyperplasia of the adrenal glands. The Clinical investigator 72: 979-984.

17. Inuzuka M, Tamura N, Sone M, Taura D, Sonoyama T, et al. (2012) A case of myelolipoma with bilateral adrenal hyperaldosteronism cured after unilateral adrenalectomy. Intern Med 51: 479-485.

18. Sukor N, Gordon RD, Ku YK, Jones M, Stowasser M (2009) Role of unilateral adrenalectomy in bilateral primary aldosteronism: a 22-year single center experience. J Clin Endocrinol Metab 94: 2437-2445. 